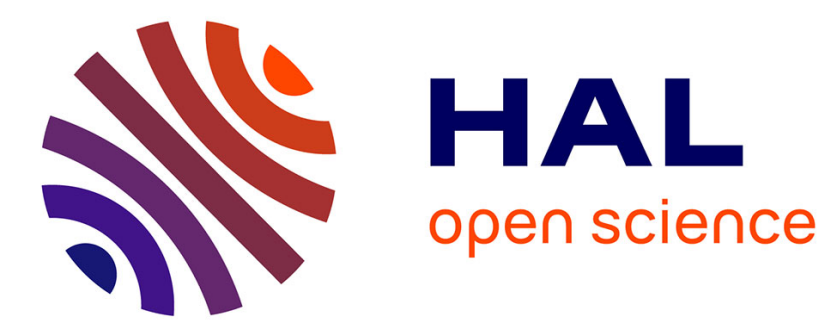

\title{
Polizei, Justiz und rassistische Diskriminierungen in Frankreich
}

\author{
Fabien Jobard, René Lévy
}

\section{To cite this version:}

Fabien Jobard, René Lévy. Polizei, Justiz und rassistische Diskriminierungen in Frankreich. Karim Fereidooni; Meral El. Rassismuskritik und Widerstandsformen, Springer VS, pp.529-544, 2017, 9783-658-14720-4. 10.1007/978-3-658-14721-1 . halshs-01351360

\section{HAL Id: halshs-01351360 https://shs.hal.science/halshs-01351360}

Submitted on 6 Sep 2016

HAL is a multi-disciplinary open access archive for the deposit and dissemination of scientific research documents, whether they are published or not. The documents may come from teaching and research institutions in France or abroad, or from public or private research centers.
L'archive ouverte pluridisciplinaire HAL, est destinée au dépôt et à la diffusion de documents scientifiques de niveau recherche, publiés ou non, émanant des établissements d'enseignement et de recherche français ou étrangers, des laboratoires publics ou privés. 


\section{Polizei, Justiz und rassistische Diskriminierungen in Frankreich $^{1}$}

Fabien Jobard

René Lévy

\section{Einleitung}

Behandelt die französische Polizei alle Personen, die ihrem Zugriff unterliegen, gleich, auch wenn diese verschiedener Herkunft sind? Praktiziert die Strafjustiz gegenüber Menschen verschiedener Herkunft, die ihrer Gerichtsbarkeit unterworfen sind (Opfer, Beschuldigte und Verurteilte), einen diskriminierungsfreien Umgang?

Diese Fragen haben in der öffentlichen Debatte in Frankreich an Nachdruck gewonnen, v.a. seit Beginn der 1980er Jahre, als städtische Unruhen Gewalt und Misshandlungen durch die Polizei in den Fokus der Aufmerksamkeit rückten (vgl.Zauberman/Lévy 2003). Wie so häufig hat sich die französische Gesellschaft keine besonders ausgefeilten Mittel erdacht, um die öffentliche Meinung oder das staatliche Handeln auf eine informierte Grundlage zu stellen. Die Arbeiten, die einen quantitativen Zugriff auf diese Frage bieten, lassen sich bis heute an einer Hand abzählen. Dies hängt nicht zuletzt damit zusammen, dass die Soziologie den,,repressiven Institutionen“ wie Polizei und Justiz in Frankreich schon immer einen sehr kleinen Bereich sozialwissenschaftlicher Forschung zugewiesen hat. Gleichwohl sind in den letzten Jahren - teilweise angestoßen durch unser Forschungszentrum, das CESDIP (Centre de recherchessociologiquessur le droit et les institutionspénales) - immer mehr Arbeiten zu diesen Fragen entstanden. Sie schaffen die Bedingungen für ein besseres Verständnis der besagten Phänomene.

${ }^{1}$ Dieser Aufsatz wurde zum grössten Teil von Kolja Lindner aus dem Französischen übersetzt und von den Autoren und Herausgeber_innen überprüft. 


\section{Der Forschungsstand zu Beginn der 1980er Jahre}

Die ersten Forschungen, die in Frankreich zum Thema Diskriminierungen im Bereich des Strafrechts unternommen wurden, haben alsbald festgestellt, dass männliche Ausländer überproportional mit der Justizmaschinerie in Kontakt kommen, insbesondere durch Gefängnisaufenthalte. Dafür wurden zwei Erklärungen vorgebracht. Erstenswurde auf die soziale Notlage zahlreicher Ausländer hingewiesen, die sie dazu bringen würde, sich durch Kleinkriminalität zu verdingen. Diese Form der Kriminalität auf offener Straße setze sie verstärkt der polizeilichen Überwachung aus. Zweitens hob man den Umstand hervor, dass Richter gegenüber prekarisierten und/oder ausländischen Angeklagten eher Gefängnisstrafen verhängten, und dies nicht nur, da die Verurteilten oft unfähig seien, Geldstrafen zu begleichen. Hinzu käme, dassdie Richter befürchteten, Ausländer würden in ihr Heimatland zurückkehren und sich somit der Strafverfolgung entziehen (einen Überblick bieten Costa-Lascoux/Soubiran 1980 bzw. Tournier/ Robert 1991).

Die Schwierigkeit bestand also darin, zu erfassen, ob allein das Ausländer- oder Immigranten-Sein eine überproportionale Bestrafung verursacht. Oder hatten wir es lediglich mit einer Justiz und Polizei zu tun, die zunächst die Armen verfolgt und bestraft?Die rechtswissenschaftliche Dissertation einer der beiden Autoren des vorliegenden Aufsatzes war die erste, dieser Frage gewidmete Untersuchung (vgl. Lévy 1987). Diese hatte dieFestnahmepraxen der Pariser Polizei zwischen 1979 und 1981 zum Gegenstand. Auf Basis der Akten von 538 auf öffentlichen Straßen festgenommenen Personen wurden dabei die von den Polizisten selbst benutzten Kriterien analysiert (,europäischer“, „nordafrikanischer“ oder „afrikanischer Typ“).Es konnte gezeigt werden, dass die Variable „,nordafrikanisch“ aussagekräftiger war, als alle anderen Variablen; aussagekräftiger als beispielsweise dersoziale Status (Beschäftigung und Familiensituation) oder mögliche Einträge im Vorstrafregister. In einer parallel erfolgten Beobachtung der Polizeiarbeitwurdenüberproportional vieleAusweiskontrollen von Nordafrikanern festgestellt. Zudem konnte gezeigt werden, dass die ethnische Zusammensetzung derer, die letztlich der Justiz überstellt wurden, nicht identisch war mit denjenigenaller derer, die kontrolliert wurden. Und schließlichunterschied sich letztgenannte Gruppe in ihren Eigenschaften von der Gesamtbevölkerung. Für LévywarendieseAbweichungen sowohl in den selektiven,die betroffenen Bevölkerungsgruppen fokussierenden Polizeipraxen sowiein Vorgängen in den späteren Entscheidungsinstanzen begründet (ebd., S. 144).

Diese Forschung hat zunächst keine vergleichbaren Arbeiten nach sich gezogen. Vielmehr schien Aubusson de Cavarlay, der alle 1978 gegen 342.000 Männer 
ausgesprochenen Gerichtsurteile untersucht hatte (von denen 13 Prozent Ausländer waren, die im Allgemeinen häufiger schwerer, gewalttätiger Straftaten angeklagt waren), ein endgültiges Urteil gesprochen zu haben: „Geldstrafen treffen Bürger und Kleinbürger, Gefängnisstrafen Subproletarier und Bewährungsstrafen einfache Leute“ (Aubusson 1985, S. 293). Tatsächlich warenArbeitslose bei gleichen Vergehen, ganz egal worin diese bestanden, die soziale Gruppe, die am ehesten mit einer Gefängnisstrafe zu rechnen hatte. So entstand der Begriff der „Dauergäste“ des Strafsystems. Allerdings wirkte sich das Ausländer-Sein (wie auch der Umstand jugendlich zu sein) ebenfalls auf die Wahrscheinlichkeit aus, härter verurteilt zu werden (ebd., S. 301. vgl. auch Herpin 1977).

Die Institution der Polizei wurde zu Beginn der 1980er aufgrund ihres Umgangs mit jungen Ausländern zunehmend problematisiert. Die Unruhen im Großraum Lyon, die sog. Marche des Beurs, die sowohl durch rassistische Gewalt als auch durch Übergriffe der Polizei ausgelöst wurde, aber auch die Entstehung der ersten ethnographischen Forschungen über die Polizei haben zu eigenständigen Untersuchungen von Diskriminierungen durch die Ordnungskräfte geführt (vgl. Lindner 2009).

\section{Forschungen zum Rassismus der Polizei}

Sind Polizisten rassistisch? Unterliegt die Polizei als Institution einem bestimmten strukturellen Rassismus? Angesichts des Profils der seit den 1970er Jahren durch Polizeigewalt zu Tode Gekommenen sowie der besonderen Rolle, die die Polizei in der Repression nordafrikanischer Arbeiter während des algerischen Unabhängigkeitskrieges sowohl in Algerien selbst als auch in der französischen Metropole spielte (vgl. Blanchard 2007. 2011), stellte sich diese Frage gewissermaßen von selbst. Sich ihr anzunehmen, brauchte die universitäre Forschung allerdings einige Zeit.

\subsection{Polizeiliche Wahrnehmung}

Ausgehend vonFokusgruppen-Interviews mit Polizisten und von durch Wissenschaftler_innen organisierte Zusammentreffen zwischen Polizist_innen und Aktivist_innen antirassistischer Gruppenentwickelten Wieviorka und seine Forschungsgruppe (1992) den in dieser Formulierung ursprünglich von einem Polizisten stammenden Gedanken, „dass man in der Polizei nicht rassistisch ist, sondern es wird“ (Wieviorka et al., 1992, S. 262). Anders gesagt: es ist weniger die Persönlichkeit des Polizisten, die in diesem Zusammenhang von Bedeutung 
ist, als ein situationsbedingter Rassismus, der von den wiederholten Konfrontationen zwischen Polizisten und Ausländerbzw. Migranten zehrt. In Frankreich ist die Polizei eine zentralstaatlich organisierte Institution, die ihren Nachwuchs, nachdem dieser die Eingangsprüfung bestanden hat und ohne dass er darauf vorbereitet ist, bevorzugt in die Problemviertel städtischer Ballungsgebiete entsendet, insbesondere im Pariser Großraum. Die Konfrontation mit Bevölkerungsgruppen, die aus den ehemaligen französischen Kolonien kommen und als erstes von der Desindustrialisierung betroffen sind, führt zu diesem „situationsbedingten Rassismus“, den ein Polizist, den wir 2004 interviewt haben (Jobard 2008, 271) wie folgt zusammenfasst:

„Aber gut.... Also Rassismus... (überlegt) Früher hatte ich Freunde, die aus Nordafrika stammten, jetzt habe ich keine mehr. Weil selbst in der Polizei, verstehst du... Ich weiß nicht, ob man rassistisch ist oder ob man es wird, nur muss man sich mal anschauen, wer einem da gegenüber steht (...). Diese Kerle verbringen ihre Zeit damit, den anderen Angst zu machen, und Angst kann zu nichts Gutem führen. Die Angst hindert dich daran, auf den anderen zuzugehen, das ist einfach so, ganz automatisch. (überlegt) Als ich klein war, hätte ich mir kaum vorstellen können, dass ich mal rassistisch werde... Bin ich überhaupt rassistisch? Schwer zu sagen (...). Wenn mein bester Kumpel, ein Schwarzer, in... (sein Heimatort, eine Stadt 100 Kilometer von Paris entfernt) mich hören würde, du kannst dir ja vorstellen... Aber gut, vor ihm habe ich keine Angst, verstehst du. Er macht mir keine Angst.“

Die zuletzt unternommenen Reformen in Polizei und Justiz haben nicht wenig zur Verfestigung der polizeilichen Sichtweisen beigetragen: Indem die Justiz dazu angehalten wurde, sich aller, d.h. selbst geringer Vergehen auf offener Straße anzunehmen, haben mehrere Regierungen nacheinander den Druck auf die Polizist_innen erhöht, die Personalien einer größtmöglichen Zahl von Straftäter festzustellen, so dass sich das repressive Netz für junge Männer in sozialen Notlagen, unter denen sich eine Vielzahl von Immigranten oder Franzosen mit Migrationshintergrund befindet, noch enger geworden ist (vgl. Mucchielli 2003. Keller/Gauthier 2010.Fassin 2013). Bestimmte Delikte, die typisch für die Konfrontation zwischen Polizisten und Jugendlichen sind, haben seit Beginn der 1990er Jahre einen drastischen Anstieg erfahren: Kannabiskonsum, Beleidigung oder Widerstand gegen die Staatsgewalt (vgl. Jobard 2004). Dadurch hat sich bei den Polizisten die Wahrnehmung gefestigt, dass sich die Welt in zwei unterschiedliche Gruppen teilt: die Anständigen (gegenüber denen sich die Polizisten zunehmend indifferent verhalten) und das „Gesindel“ (ein Begriff, den der Innenminister Sarkozy 2005 verwandte). Die beiden Soziologen Lukas/Gauthier konstatierten aufgrund von Interviews mit und Beobachtungen von französischen und deutschen Polizisten, dass erstere im 
Verhältnis zu letzteren viel stärker auf „Respekt und Disziplin“ derjenigen insistierten, die ihrem Zugriff unterlagen. Zudem agierten die französischen Beamten deutlich autoritärer, bspw. durch Ausweiskontrollen (vgl. Gauthier/Lukas 2010).

Dennoch ist der Rassismus der Polizei eine komplexe Angelegenheit. Wir wissen nicht genau, ob verächtliches, unverschämtes oder diskriminierendes Verhalten Ausländer generell oder nur selektiv betrifft, d.h. nur diejenigen unter ihnen, die die Polizisten zu Recht oder Unrecht verdächtigen, zur kriminellen Klientel zu gehören.

In regelmäßigen Abständen sind mittels Umfragen die Einstellungen von Polizisten zu ermitteln versucht worden. In der ersten dieser Untersuchungen wurden 1982 alle etwa 100.000 französischen Polizeibeamten befragt (vgl. Hauser/Masingue 1982). Zudem haben die Soziologen Monjardet/Gorgeon eine Kohortenanalyse mit 1.167 Rekruten des 121. Jahrgangs der Polizeiakademie zu verschiedenen Momenten ihrer beruflichen Laufbahn zwischen 1992 und 2002 durchgeführt (vgl. Gorgeon/Monjardet 2004. 2005). Schließlich wurden mit einer weiterensoziodemographischenUntersuchung, die auf die von Hauser und Masingue antwortete, $2003 \quad 5.221$ Polizisten aller Dienstgrade und Zuständigkeitsbereiche beforscht(vgl. Pruvost 2009. Pruvost/Roharik 2011). Auf Basis all dieser Analysen kann die Hypothese einer Abnahme fremdenfeindlicher Einstellungen bei den verschiedenen Generationen von Polizisten aber auch im Verlauf einzelner Laufbahnen aufgestellt werden.

In der Untersuchung von 1982 gaben 15 Prozent der befragten Polizist_innen an, bevorzugt auf Immigranten zu achten, als dritte Gruppe nach Jugendlichen und Drogendealern. Die soziodemographische Untersuchung von 2003 stellte ebenfalls die Frage nach Aufmerksamkeitsökonomien. Zwei besondere Entwicklungen konnten dabei verzeichnet werden: Immigranten werden nunmehr nur noch von neun Prozent der Polizist_innengenannt (sie belegen den fünften Platz unter den Problemgruppen) und die Einstellungen der Streifenpolizisten können nicht mehr von denen der Kommissare unterschieden werden. Diese Ergebnisse werden von der Kohortenanalyse von Gorgeon und Monjardet untermauert. Auf die Gründe für Kriminalität befragt, landet „,der Migranten-Zustrom“ bei Polizisten am Beginn ihrer Laufbahn auf dem dritten Platz (13 Prozent), weit hinter ökonomischen und sozialen Faktoren (59 Prozent). Zehn Jahre später landet Einwanderung bei zehn Prozent, ökonomische und soziale Faktoren nennen 15 Prozent als ausschlaggebend. Die Nachlässigkeit der Justiz erreicht dagegen45 Prozent - trotz des regelmäßigen Aufrufs von Politiker_innen,insbesondere irreguläre Einwanderung zu bekämpfen (vgl. Fischer/Darley 2010). 


\section{Das Verhalten der Polizist_innen}

Der Umgang der Polizist_innen mit Immigrant_innen oder ihren Nachkommen wurde bisher in zweierlei Hinsicht quantifiziert: bezüglich der Prozesse innerhalb der Polizei und bezüglich des Verhaltens der Polizisten gegenüberPersonen, die nicht ihrer Berufsgruppe angehören.

\subsection{Diskriminierungen innerhalb der Polizei in Deutschland und Frankreich}

In welcher Situation befinden sich Minderheiten innerhalb der Polizei? In Deutschland liegt Schätzungen zufolge der Anteil der Polizeibeamtenmit Migrationhintergrund bei ein bis drei Prozent (vgl. Hunold et al. 2010). Mitte der 1990er Jahre fing in Frankreich die Politik an, den Aufbau einer - um das Bonmot des französischen InnenministersChevènement von 1997 zu bemühen „Polizei nach dem Bild der Bevölkerung“ zu forcieren. Der Berufsstand wurde nach den etwas deutlicheren Worten des französischen Premierminister Lionel Jospin von 1999 ,den Jugendlichen aus den (unterprivilegierten) Problemvierteln und jenen mit Migrationshintergrund“" geöffnet. Die Auswirkungen zahlreicher Maßnahmen sind keinesfalls zu unterschätzen. Nach einer Studie, die in den Jahren 2008 und 2009 im Innenministerium durchgeführt wurde, liegt der Prozentsatz von Beamten (vor allem Polizisten) mit Migrationshintergrund bzw. aus den französischen Überseegebieten bei 20 Prozent. Es muss aber hier unterschieden werden, da auch der Begriff ,mit Migrationshintergrund“ genauer präzisiert werden muss. Unter diesen Beamten ist der Prozentsatz derer, die im Ausland geboren wurden, mit 1,4 Prozent gering; jener der Beamten, deren Eltern in Afrika bzw. aus anderen Teilen der Welt geboren sind, liegt bei 3,4 bzw. bei 6,7 Prozent (vgl. Meurs/Héran 2009).

Als Resultat der politischen Anstrengungen ist ein deutlich höherer Anteil von Menschen mit Migrationshintergrund in den Polizist_innenkohorten nach 1997 zu verzeichnen: Der Anteil der Personen, deren Eltern auf dem afrikanischen Kontinent geboren wurden, lag zwischen 1990 und 1996 bei zwei Prozent und stieg ab 1997 auf sechs Prozent. Die von Meurs und Héran aufgeworfenen Fragen nach der Wahrnehmung des Arbeitsklimas, den Verhältnissen zu den Vorgesetzten usw. lassen keinen signifikanten Unterschied zwischen den Gruppen verschiedener Herkunft erkennen - mit Ausnahme der Angriffe, denen die Beamten in der Öffentlichkeit ausgesetzt waren. Diese wurde von den Polizisten mit Migrationshintergrund oder aus den Überseegebieten als stärker empfunden (wobei dies vermutlich darauf zurückgeht, dass die Betroffenen 
durchschnittlich jünger sind und daher in den härtesten Stadtteilen eingesetzt werden).

Die Polizei ändert sich also langsam, aber sie tut eszweifellos - auch ungeachtet der starken institutionellen Widerstände, wie es Duprez/Pinet in ihrer Arbeit über die Aufnahmeprüfung für die Polizei gezeigt haben.Ihre Untersuchung illustriert zunächst die sicher geringe, aber nicht zu vernachlässigende Zahl von Bewerbern mit nordafrikanischem Migrationshintergrund, die durch eine Analyse der auf den Bewerbungsbögen angegebenen Familiennamen identifiziert wurden: von 6,5 bis elf Prozent je nach Stadt (vgl. Duprez 2010). Allerdings ist die Erfolgsquote bei den Bewerbern mit nordafrikanischem Migrationshintergrund im Vergleich zu den anderen Bewerbern um 3,4 Prozent geringer (8,6 gegen 12 Prozent der Bewerber_innen). Die beiden Wissenschaftler haben die mündlichen Prüfungen und die Beratungen der Auswahlkommissionen beobachtet. Dabei konnten sie zweifelsfrei diskriminierendes Verhalten der Prüfer_innen feststellen, insofern die Bewerber_innenmit nordafrikanischem Migrationshintergrund, insbesondere jene Männer, die aus Sozialbausiedlungen kamen, Fragen beantworten mussten, die anderen Bewerbern (weder den anderen Männern noch den Frauen, gleich welcher Herkunft) nicht gestellt wurden. Den Betroffenen wurden beispielsweise Fragen wie „Wenn dein Cousin einen deiner Kollegen bedroht, ziehst du dann deine Waffe?“, „Was machst du, wenn du deinen Bruder festnehmen musst?" usw. gestellt.

Der institutionelle Widerstand manifestiert sich während der Aufnahmeprüfungen und verschwindet keinesfalls in der weiteren Laufbahn der Betroffenen, wie Gauthier (2012) in seiner Dissertation klar gezeigt hat. Ausgehend von Beobachtungen und etwa sechzig Interviews mit Polizisten aus Berlin und Pariser Vorstädten, unter denen sich etwa fünfzehn Beamte mit Migrationshintergrund befanden, arbeitet Gauthier heraus, dass diese Polizisten von ihren jeweiligen Dienststellen Situationen ausgesetzt werden, in denen ,sie sich beweisen müssen“. Die Betroffenen „entwickeln ein ganzes Repertoire an Taktiken, mit denen sie ihren Platz und ihre Legitimität im spannungsgeladenen Zusammenhang mit Ihresgleichen sicherzustellen versuchen" (Gauthier 2011, S. 476). Indessen reagieren die Institutionen beider Länder unterschiedlich. In Frankreich wird die Minderheitenproblematik verschwiegen und damit werden auch die Konflikte, die durch sie entstehen (und meistens auf Kosten der Polizisten aus Minderheiten gelöst werden). Im Kontrast dazu wird in Berlin Differenz hervorgehoben und aufgewertet: Sie wird eine eigene polizeiliche Kompetenz, ,interkulturelle Kompetenz“ genannt. Diese kann die Ressourcen der Polizei in ihrem städtischen Umfeld erhöhen und helfen, Spannungen besser $\mathrm{zu}$ objektivieren und zu handhaben. So wird die Situation der Minderheiten 
Gegenstand einer eigenen Politik der Polizeibehörden, die sowohl nach Innen als auch nach Außen Ergebnisse zeigt.

\subsection{Identitätskontrollen in Deutschland und Frankreich}

Das mit am meisten umstrittene polizeiliche Vorgehen gegen migrantische Jugendliche sind die Identitätskontrollen. Anders als in den meisten deutschen Bundesländern kann die Polizei (und die Gendarmerie) in Frankreich verdachtsunabhängige Kontrollen durchführen, vorausgesetzt der sie vornehmende Polizist ist in der Lage, ein halbwegs gesetzeskonformes Verlaufsprotokoll zu schreiben. Die entsprechenden Unterschiede zwischen den beiden Ländern lassen sich an den Ergebnissen desEuropean Union Minorities and Discrimination Survey (EU-MIDIS) ablesen. Mit dieser Untersuchung hat die Europäische Agentur für Grundrechte aufgrund von Umfragen unter den Betroffenen 2010 festhalten können, dass in Frankreich rund 40 Prozent der Menschen mit nordafrikanischem Aussehen in den letzten zwölf Monaten durch die Polizei kontrolliert wurden, wohingegen in Deutschland nur 25 Prozent der Menschen mit türkischer Herkunft angaben, eine solche Kontrolle erlitten zu haben (vgl. European Union Agency for Fundamental Rights 2010). Die den Polizisten gegebene Macht der Identitätskontrolle ist in Frankreich seit mindestens drei Jahrzehnt stark umstritten. In einem Artikel, den die Berliner Zeitung kurz nach den Revolten von 2005 veröffentlichte, halten die französischen Soziologen Mucchielli/Lapeyronnie (2005, S. 32) fest: „Wenn fast ausnahmslos 'weiße' Polizisten in eine Bevölkerung eingreifen, die sie nicht kennen, wenn sie irgendwie die herausgreifen, die ihnen 'verdächtig' scheinen (also fast immer Schwarze und Arabisch-stämmige) und dabei dieselbe Gewaltbereitschaft in Wort und Tat beweisen wie die Straftäter, die sie verfolgen wollen, dann muss man sich nicht wundern, wenn das als Zeichen von Unterdrückung und Rassismus gesehen wird“.

In Zusammenarbeit mit unserem amerikanischen Kollegen John Lamberth haben wir versucht, den diskriminierenden Charakter dieses Vorgehens zu evaluieren (vgl. Jobard et al. 2013. Jobard/Lévy 2013).Es wurden fünf unterschiedliche Pariser Orte ausgewählt, an denen üblicherweise viele Identitätskontrollen vorgenommen werden (drei Orte rund um den Nordbahnhof, zwei im Pariser Zentrum). In einer ersten Phase wurden die Charakteristika von allen Passanten aufgenommen, damit die Kontrollierten mit der anwesenden Gesamtbevölkerung verglichen werden konnten. Fünf verschiedene Variablen wurden definiert, die sich alle auf das äußere Erscheinungsbild bezogen: mutmaßliches Alter (jung/nicht jung), mutmaßliches Geschlecht, mutmaßliche Herkunft (Weiße/Schwarze/Maghrebiner/Inder bzw. Pakistani/andere Asiaten/sonsti- 
ge/unbekannt), Bekleidung bzw. Ausstaffierung (Freizeitkleidung/Business/Jugendkultur), mitgeführtes Gepäck (kein oder ein großes Gepäckstück/normale Tasche). Auf diese Weise wurden insgesamt an den fünf Orten die Merkmale von über 37.000 Personen erhoben. In einer zweiten Phase folgten diejenigen, die die Daten erhoben, Polizeibeamten ohne deren Wissen, um die von ihnen durchgeführten Kontrollen zu erfassen. Unsere Mitarbeiter_innen waren mit Mobiltelefonen ausgestattet, mithilfe derer sie die Charakteristika der Kontrollierten aufnahmen und sie dann per SMS in die USA schickten, wo ein Server sie in statistische Daten umwandelte. Nach einem knappen halben Jahr um den Jahreswechsel 2007/2008 hatten wir 525 Kontrollen erfasst.

Als erstes Resultat konnte verzeichnet werden, dass sich die kontrollierte Bevölkerungsgruppe in ihrer Zusammensetzung radikal von der für Kontrollen zur Verfügung stehenden Gesamtbevölkerung an dengewählten Ortenunterscheidet. In der Gruppe der Kontrollierten sind ,sichtbare Minderheiten“ überrepräsentiert. An Orten, an denen People of Colornicht sehr zahlreich sind, wie am Ausgang des Thalys, des Schnellzuges zwischen Paris und Brüssel bzw. Amsterdam (weniger als 15 Prozent), werden sie überdurchschnittlich häufig kontrolliert (sie machen fast die Hälfte der Kontrollierten aus). Aber auch dort, wo sich viele Nicht-Weiße aufhalten, etwa in den Gängen, die am Gare du Nord zu den Regionalzügen der Vorstädte führen (57 Prozent der Anwesenden), gilt ihnen überdurchschnittliches polizeiliches Interesse (80 Prozent der Kontrollierten).

Aber dieser Unterschied zwischen den Kontrollierten und der Gesamtbevölkerung an den jeweiligen Orten ist nicht nur bezüglich Herkunft und Hautfarbe offensichtlich. Er zeigt sich auch bei allen anderen Variablen. Die Polizisten kontrollieren häufiger junge Männer (zwischen 3und 100 mal häufiger als ältere Männer), Männer überhaupt (zwischen 1,5 und 10mal mehr als Frauen), typisch jugendlich gekleidete Passanten (zwischen fünf und 16 mal mehr als Leute ohne besondere Merkmale), und selbst Passanten ohne Taschen geraten öfter in den Fokus der Polizei. Die Frage lautete nun, ob eine dieser Variablen signifikant bestimmender war als andere und insbesondere, ob das Kriterium der Hautfarbe oder der unterstellten Herkunft bei der Entscheidung der Polizisten, eine Person zu kontrollieren, überwog. Die Antwort darauf ist ausgesprochen schwierig, denn die Variablen sind in Wirklichkeit alle miteinander verbunden: Sowohl Hautfarbe als auch typisch jugendlicher Kleidungsstil motivieren die Kontrollentscheidung. Beide Variablen sind eng miteinander korreliert und es ist unmöglich, sie in ihrer jeweils eigenen Wirkung zu isolieren (vgl. Jobard et al. 2013 für eine ausführlichere Analyse). 
Die Identitätskontrolle illustriert daher ziemlich gut die Zweideutigkeit des Begriffs des polizeilichen Rassismus: Dieser macht sich je nach Fall mehr oder wenigeran der Hautfarbe oder an anderen Zeichen der polizeilichen Semantik fest, wie etwa dem Kleidungsstil, der auf die Herkunft aus einer schwierigen Vorstadt hinweisen kann. Diese zweideutigen Resultate werden durch eine demnächst erscheinende Studie gestützt, die eine repräsentative Gruppe von Studierenden in Paris und im Großraum der französischen Hauptstadt untersucht hat (vgl. Jounin et al. 2015). 2.400 Studierende wurden im Frühjahr 2012 befragt, wie oft sie Polizeikontrollen erleiden mussten. Dabei wurde den Studierenden die Möglichkeit gegeben, ihre Herkunft selbst zu bestimmten. Die Gesamtgruppe setzte sich aus 56,4 Prozent Weißen, 37,4 Prozent Nicht-Weißen und 6,2 Prozent Studierenden, die keine entsprechenden Angaben machen wollten, zusammen.

Eine erste Auskunft, die aus der Studie gewonnen werden kann, besteht darin, dass die (erklärte) Wahrscheinlichkeit, von der Polizei kontrolliert zu werden, zwischen nicht-weißen und weißen Männern nicht signifikant variiert: 37,7 Prozent Ersterer geben an, in den letzten zwölf Monaten kontrolliert worden zu sein, gegenüber 42,9 Prozent der Letztgenannten. Allerdings sind bei den NichtWeißen Mehrfachkontrollen häufiger (56 Prozent gegen 41,4 Prozent geben an mehrfach kontrolliert worden zu sein). Zudem wird die Polizeikontrolle in den verschiedenen Gruppen unterschiedlich erlebt: 46 Prozent der nicht-weißen Männer geben an, Menschen in ihrem Umfeld $\mathrm{zu}$ haben, die regelmäßig kontrolliert würden (gegenüber 35 Prozent der Weißen). Zudem geben 19 Prozent der nicht-weißen Männer an, aufgrund von Polizeikontrollen ihre Wege (gegenüber 14 Prozent), ihr Verhalten (28 gegenüber 22 Prozent) und ihr Aussehen, v.a. ihren Kleidungsstil (16 gegenüber 8Prozent) zu ändern. Tatsächlich scheint der studentische Lebensstil ein Faktor zu sein, der die Wahrscheinlichkeit einer Kontrolle erhöht.

So ist statistisch auffällig, dass Studierende, die ein Auto nutzen, häufiger kontrolliert werden als andere. Dies gilt auch für Studierende, die mehr als zwei Stunden pro Tag in öffentlichen Transportmitteln verbringen, die mehr als einmal die Woche abends ausgehen, die in Clubs gehen und die Kannabis besitzen. Diese mit den Lebensgewohnheiten verbundenen Variablen erklären, warum Nicht-Weiße angeben, nicht häufiger als Weiße kontrolliert zu werden. Denn letztere bewegen sich häufiger in Autos fort, gehen häufiger abends aus, v.a. in Clubs, und sind häufiger im Besitz von Kannabis. Die Nicht-Weißen sind besonnener, ob aus Angst vor der Polizei oder aufgrund geringerer finanzieller Ressourcen. $\mathrm{Zu}$ diesen Variablen des Lebensstils muss man, wie wir es in unserer Studie gemacht haben, auf differenzierte Weise das Aussehen hinzufügen. 76 Prozent der Träger eines Basecaps wurden kontrolliert, aber nur 
42 Prozent derer, die keine solche Kopfbedeckung trugen. Zwei weitere Unterschiede im Aussehen sind entscheidend, nämlich Jogginghose (58,5 gegen 41,5 Prozent) und Kapuze (53 gegen 42,5 Prozent). Andere Charakteristika fallen nicht ins Gewicht, etwa geschorene Haare, Bart oder Dreadlocks. Das Kontrollrisiko jener, die ein Basecap, eine Jogginghose und eine Kapuze tragen, ist fünf mal höher als das jener, die keines der drei Bekleidungsstücke aufweisen. Nun tragen allerdings die Nicht-Weißen häufiger eines (65,5 gegen 51,5 Prozent) oder mehrere dieser Kleidungsstücke (19 gegen elf Prozent).

Die umfragegestützte Untersuchung von Jounin und seinen Kollegen bestätigt Unsere. Sie weißt auf die Existenz einer ,polizeilichen Klientel“ (Jobard 2008, 270) hin: eine Personnengruppe, die aufgrund bestimmter Attribute (Geschlecht, Rasse und Kleidungsstil) im Zentrum polizeilicher Aufmerksamkeit steht.Zusammengenommen mit ökonomischen Aspekten beeinflusst diese Aufmerksamkeit die sozialen Praktiken dieses Klientels, das seine Ausflüge in die Stadt verringert, um den Polizeikontrollen zu entgehen (was wiederum die Wahrscheinlichkeit einer Kontrolle verringert).

\section{Diskriminierungen und Strafjustiz}

Ist die Strafjustiz blind gegenüber unterschiedlicher Herkunft? Verschiedene Forschungen haben zuletzt den Wissensstand zur Beantwortung dieser Frage vergrößert.

Vor gut zehn Jahren hat Pager (2008) gezeigt, dass die französischen Gerichte, von denen die meisten „harten“ Strafen ausgesprochen werden (Untersuchungshaft, Meldeauflagen und Gefängnisstrafen), sich in den Departements befinden, die die größte Konzentration junger Männer mit nordafrikanischem Migrationshintergrund aufweisen. Allerdings wurde dabei kein Zusammenhang mit anderen, gesellschaftlichen Variablen hergestellt (Anzahl der Ausländer bzw. der ausländischen Jugendlichen, Arbeitslosenquote, gezählte rassistische Vorfälle, Anzahl der Departementbewohner und vor allem registrierte Straftaten), so dass die Variable ,junge Männer mit nordafrikanischem Migrationshintergrund" besonders heraus stach.

Ebenfalls zu dieser Zeit hat eine Studie der CIMADE, einer Organisation, die Asylsuchende unterstützt, herausgestellt, dass die härtesten Strafen Ausländer treffen (vgl. CIMADE 2004). So beobachteten die Mitglieder von CIMADE 2002382 Verfahren vor einem Gericht in Montpellier und zeigten, dass 30 Prozent der verurteilten Franzosen und 43 Prozent der Ausländer eine Gefängnisstrafe verbüßen mussten. Auch bei Berücksichtigung der Vergangenheit der Betroffenen ließ sich diese Ungleichheit feststellen, denn 47 
Prozent der Ausländer mit Eintrag ins Vorstrafenregister (gegenüber 35 Prozent der französischen Staatsbürger) und 38 Prozent der Ausländer ohne Eintrag ins Vorstrafenregister (gegenüber 25 Prozent der französischen Staatsbürger) mussten eine Freiheitsstrafe verbüßen. Die CIMADE verzeichnete zudem, dass Ausländer unter den arbeitslosen Häftlingen überrepräsentiert waren. Leider unterließt die Organisation es aber die beiden Variablen (Staatsangehörigkeit und Beschäftigungsstatus) miteinander zu kreuzen.

Kürzlich hat Léonard (2010) in seiner Dissertation zu messen versucht, ob Ausländer, die sich in der gleichen sozialen Lage wie französische Staatsbürger befinden, in Schnellverfahren [comparutionimmédiate] härtere Strafen bekommen.Von Gerichten in Lille und Lyon erhielten 67 Prozent der Franzosen und 77 Prozent der Ausländer Freiheitsstrafen. Bei zwei kleineren Kammern lagen die Anteile sogar bei 86 und 90 Prozent. Bei dieser Art Schnellverfahren, die von Staatsanwälten eingeleitet werden, sind Gefängnisstrafen sehr häufig. Léonard beobachtet nun allerdings, dass Staatsanwälte Schnellverfahren einleiten, wenn die Angeklagten in der Vergangenheit bereits einmal verurteilt wurden - außer bei Ausländern, die bevorzugt in Schnellverfahren verurteilt werden, gleich welchen strafrechtlichen Hintergrund sie mitbringen. Während sich unter den verurteilten französischen Staatsbürgern vor allem solche finden, die bereits zuvor von der Justiz belangt wurden, stehen in der Gruppe der Ausländer diejenigen mit Erfahrungen mit der Justiz denen gegenüber, die sich zum ersten Mal vor Gericht befinden. Wie anderswo in Europa ist die Rolle der Staatsanwälte bei der Ausrichtung der Strafverfahren in Frankreich von großer Bedeutung (vgl. Jehle/Wade 2004). Hier liegt ein diskriminierendes Moment. Wie eine ganze Reihe von Arbeiten vor ihm verzeichnet Léonard allerdings, dass die Angeklagten, die einer Beschäftigung nachgehen, weniger häufig zu einer Gefängnisstrafe verurteilt werden. Dennoch werden die ausländischen Angeklagten, die weder einer Beschäftigung nachgehen, noch im Vorstrafenregister verzeichnet sind, häufiger zu Gefängnisstrafen verurteilt als Franzosen mit dem gleichen Profil (80 gegenüber 54 Prozent).

Auch wir haben in einer mit Sophie Névanen durchgeführten Forschung die Bedeutung der gewählten Art des Strafverfahrens nicht nur für die ausländischen Angeklagten, sondern für diese und die Angeklagten mit Migrationshintergrund, die wir durch ihre Vor- und Nachnamen sowie ihren Geburtsort identifiziert haben, nachweisen können (vgl. Jobard/Névanen 2010). Dabei wurden die Fälle von 864 vor einer Pariser Kammer wegen Widerstandes gegen die Staatsgewalt (meist Polizeibeamte) Verurteilter analysiert (zum Kontext vgl. auch Jobard 2004). In unserem Sample ließen sich 62,2 Prozent der Angeklagten der Gruppe „Europäer“, 20,1 Prozent der Gruppe „Nordafrikaner“ und 15,5 Prozent der Gruppe „Schwarze“ (und 2,2 Prozent einer Restgruppe) zuordnen. Betrachtet 
man die Gesamtheit der Angeklagten lässt sich eine deutliche Ungleichverteilung hinsichtlich der verhängten Gefängnisstrafen ohne Bewährung bei den verschiedenen Angeklagten Gruppen feststellen. Während gegen die Angeklagten der Gruppe „Europäer“ in 13,6 Prozent der Fälle Haftstrafen ohne Bewährung verhängt wurden, waren es bei den Angeklagten der Gruppe „Nordafrikaner“ 23,6 Prozent und bei der Gruppe „Schwarze“ 25,4 Prozent. Anders ausgedrückt heißt das, dass das Risiko zu einer Haftstrafe ohne Bewährung verurteilt zu werden für Nordafrikaner und Schwarze fast doppelt so hoch ist wie für Angeklagte europäischer Herkunft.

Die Datenanalyse zeigt, dass gegen Nicht-Europäer häufiger aufgrund schwererStraftaten verhandelt wird, sie häufiger als Wiederholungstäter angeklagt sind (was die voraussichtliche Strafe automatisch erhöht) und sie sich häufiger der Verhandlung entziehen bzw. nicht zu dieser erscheinen. Eine Analyse, die die Vielzahl von Faktoren in Rechnung stellt,ergibt, dass die Variable „Herkunft“ in der richterlichen Entscheidung keine eigenständige Rolle spielt. Allerdings kommt dazu die Polizei ins Spiel. Mithin durch Politik und Gewerkschaften motiviert, treten Polizisten regelmäßig als Nebenkläger auf. Dies geschieht allerdings nicht bei allen Gruppen von Angeklagten in gleichem Maße. Während bei nur 37 Prozent der Verfahren gegen Europäer Polizisten als Nebenkläger auftreten, sind es 51 Prozent bei den Verfahren gegen Nordafrikaner und 46 Prozent bei denen gegen Schwarze. Eine Regressionsanalyse zeigt, dass hier Herkunft eine klare Rolle spielt.

Safi/Simon (2013, S. 246) halten zu recht fest, „dass die Messung von Diskriminierungen eine politisch sensible und methodisch komplexe Angelegenheit ist". In Frankreich wurde die Entwicklung des im vorliegenden Aufsatz dargestellten Forschungsfeldes lange durch eine politische (und rechtliche) Kultur behindert, in der der Gebrauch „ethnischer“ oder „rassischer“ Kategorien, die die kollektive Zugehörigkeit von Individuen zum Ausdruck bringen, verpönt ist (Schnapper 2008). In der universitären Forschung wurde heftig über die Legitimität und die Zuschneidung von Kriterien gestritten (vgl. Simon 2008. CARSED 2009. COMEDD 2010. Le Bras 2010). Der Gedanke, dass man diskriminierende Kategorien selbst aufgreifen muss, um Diskriminierungen zu erfassen, hatte lange Zeit Schwierigkeiten, sich gegen diejenigen durchzusetzen, die darin eine Legitimation des Rassismus sahen. Diese Auseinandersetzung ist heute glücklicher Weise zugunsten einer sachlicheren Debatte über Daten und Methoden in den Hintergrund getreten.

Daher sind in Frankreich in den letzten Jahren vielfältige Forschungen über Diskriminierungen im Bereich des Strafrechts (Polizei und Justiz) entstanden, die systematischer sind als in Deutschland, wo es kaum quantitative und qualitative Untersuchungen gibt. Die besagten Forschungen haben vor allem 
versucht, bereits bestehenden, qualitativen Zeugnissen (aus der Presse, von Verbänden oder aus ethnographischen Untersuchungen), die allesamt ein für Minderheiten besonders nachteiliges Agieren der Polizei herausstellten, Zahlen und Quantifizierungen beiseite zu stellen. Unter diesem Blickwinkel haben die Untersuchungen der letzten Jahre den Wissensstand in vier Bereichen vermehrt. Erstens haben sie die Diskussion mit Zahlen unterfüttert, auch wenn wir in Frankreich ebenso wenig wie in Deutschland über offizielle Statistiken über „rassische“ oder „ethnische“ Zugehörigkeiten verfügen. Zweitens haben uns die besagten Arbeiten mit Informationen über die Justiz, wie bisher bereits in sehr detaillierter Weise geschehen über die Polizei versorgt.Schließlich haben die Untersuchungen unser Wissen in zwei weiteren, sehr umkämpften Bereichen vergrößert. Der erste ist jener der ,postkolonialen“ Dimension der konstatierten Diskriminierungen und Ungleichheitslagen: Hat die Stigmatisierung mit dem (durchmischten und vielfältigen) Vermächtnis des Kolonialismus zu tun? Der zweite Bereich ist eng mit dem ersten verwoben und hängt mit dem Begriff der „polizeilichen Klientel“ zusammen: Ziehen die jungen Männer, auf die sich die Polizei eingeschossen zu haben scheint, den Verdacht und die Gewalt der Polizei sowie die Härte der Justiz auf sich, weil sie ethnischen Minderheiten angehören? Oder ist hier vielmehr eine Vielzahl von Faktoren ausschlaggebend, die über die Frage der Hautfarbe und damit des Rassismus hinausgehen? Die sich aus diesen beiden Bereichen ergebenden Fragen stehen heute im Zentrum der französischen Forschungsdiskussion.

\section{Literatur}

Aubusson de Cavarlay, Bruno (1985): Hommes, peines et infractions: La légalité de l'inégalité. In: L'Année sociologique, 35, S. 275-309.

Blanchard, Emmanuel (2007): L'encadrement des Algériens de Paris (1944-1954), entre contraintesjuridiques et arbitraire policier. In:Crime, Histoire \&Sociétés/Crime, History\&Societies, 11, 1, S. 5-25.

Blanchard, Emmanuel (2011):La police parisienne et les Algériens, 1944-1962. Paris: Nouveau Monde Editions.

BürgerrechteundPolizei (2014):Racial Profiling, Nr. 104.

CARSED (2009): Le retour de la race. Contre les « statistiquesethniques ». Paris: L'Aube.

COMEDD (2010):Inégalités et discriminations. Pour un usage critique et responsable de l'outilstatistique. Paris: La Documentation française.

Costa-Lascoux, Jacqueline/Soubiran, Françoise (1980): Actualités bibliographiques: Travailleurs immigrés et contrôle social. In:Déviance et société, 4(3), S. 279-301.

Duprez, Dominique (2010): Die Personalauswahl der französischenPolizeiunterdemAspektvonHerkunftundGeschlecht. In: SozialeProbleme, 21, S. 42-72.

Fassin, Didier (2013): Die moralischeArbeit der Polizei. In: WestEnd. NeueZeitschriftfürSozialforschung, 13, 1, S.102-110. 
Fischer, Nicolas/Darley, Mathilde (2010): Managing immigration: Between an administration logic and a criminalitylogic". In: Champpénal/Penalfield, 7,

http://champpenal.revues.org.gate3.inist.fr/8005 [LetzterZugriff: 5.6.14].

European Union Agency for FundamentalRights(http://fra.europa.eu/eu-midis) (2009):EU-MIDIS

Technical Report. Methodology, Sampling and Fieldwork.Luxembourg, Office des publications de l'Unioneuropéenne, http://fra.europa.eu/sites/default/files/eu-midis_technical_report.pdf

European Union Agency for FundamentalRights (2010a):EU-MIDIS Data in Focus Report 4: Police Stops and Minorities.Luxembourg, Office des publications de l'Unioneuropéenne, http://fra.europa.eu/en/publication/2010/eu-midis-data-focus-report-4-police-stops-andminorities

European Union Agency for FundamentalRights(2010b):Towards More Effective Policing, Understanding and preventingdiscriminatoryethnicprofiling: A guide, Luxembourg, Office des publications de l'Unioneuropéenne, http://fra.europa.eu/en/publication/2012/towards-moreeffective-policing-understanding-and-preventing-discriminatory-ethnic

Gauthier, Jérémie (2011): Des corps étrange(r)s dans la police ? Les policiersminoritaires à Paris et à Berlin. In: Sociologie du travail, 53(4), S. 460-477.

Gauthier, Jérémie/Lukas, Tim (2011): Warumkontrolliert die Polizei (nicht)? UnterschiedeimHandlungsrepertoiredeutscherundfranzösischerPolizisten. In: SozialeProbleme, 2, 22,S. 174-206

Hauser, G./Masingue, B.(1982): Les policiers, leurs métiers, leur formation. Paris: La Documentation française.

Herpin, Nicolas (1977):L'application de la loi: deuxpoids, deuxmesures. Paris: Seuil.

Hunold, Daniela et al. (Hg.) (2010):FremdealsOrdnungshüter? Die Polizei in der ZuwanderungsgesellschaftDeutschland. Wiesbaden: VS Verlag.

Jehle, Jörg-Martin/Wade, Marianne (2004):CopingwithOverloadedCriminal Justice Systems. Berlin: Springer.

Jobard, Fabien (2004): Der Ort der Politik.

PolitischeMobilisierungzwischenAufstandsversuchungundStaatsgewalt in einerPariserVorstadt. In: Berliner Journal fürSoziologie, 3,S. 319-338.

Jobard, Fabien

(2008):EthnizitätundRassismusindergesellschaftlichenKonstruktiondergefährlichenKlassen.Poliz eikultur-und-praxisindenfranzösischenVororten. In: SchweizerischeZeitschriftfür Soziologie,34,2,S. 261-280.

Jobard, Fabien/Lévy, René (2013): Identitätskontrollen in Frankreich. Diskriminierungfestgestellt, Reformausgeschlossen? In:Bürgerrechte\& Polizei,104, S. 29-37.

Jobard, Fabien/Lévy, René/Lamberth, John/Névanen, Sophie (2013): Measuringappareance-based discrimination. An analysis of identitychecks in Paris. In:Population-E, 67, 3,S. 349-376.

Jounin, Nicolas et al. (2015): Le faciès du contrôle. Contrôlesd'identités, apparence et modes de vie des étudiant-e-s en Ile-de-France. In:Déviance et Société, 39, 1, S. 3-30.

Keller, Carsten/Gauthier, Jérémie (2010): KontrollpolitikundKonflikteskalation in benachteiligteStadtteilen. Eindeutsch-französischerVergleich. In: Soeffner, H-.G (Hg.):UnsichereZeiten. HerausforderungengesellschaftlicherTransformationen. Verhandlungen des 34. Kongresses der DeutschenGesellschaftfürSoziologie in Jena 2008, Wiesbaden: VSVerlag (cd-rom).

Lahalle, Annina (1980): Les rapports de police, les enquêtes des servicessociaux et les décisions des juges“, in: Les jeunesimmigrés. Eux et nous. Vaucresson: CFRES,S. 87-133.

Le Bras, Hervé (2010):Statistiques ethniques. Le vrai débat. Paris : Fondation Jean Jaurès.

Léonard, Thomas (2010): Cespapiers qui font le jugement. In: Champpénal / Penalfield, nouvelle revue internationale de criminologie, Vol. VII, http://champpenal.revues.org/7879 [LetzterZugriff: 27.9.10]. 
Léonard, Thomas (2014): De la «politiquepublique » à la pratique des comparutionsimmédiates. Unesociologie de l'actionpublique au prisme des configurations locales et nationale.Diss. Politikwissenschaft, Université Lille 2.

Lévy, René(1987):Du suspect au coupable. Le travail de police judiciaire.Genf/Paris : MéridiensKlincksieck/Médecine\&Hygiène).

Lindner, Kolja (2009): 25 Jahre 'Marche des Beurs': Kämpfe der Migration imFrankreich der 1980er Jahrenundheute. In: Peripherie. ZeitschriftfürPolitikundÖkonomie in der DrittenWelt, 2/2009, S. 304-324.

Meurs, Dominique/Héran, François (2009):Diversité et parité au ministère de l'intérieur. Rapport final. Paris: INED.

Monjardet, Dominique/Gorgeon, Catherine (2005): La culture professionnelle des policiers, uneanalyselongitudinale. In: Les cahiers de la sécuritéintérieure, 56(1), S. 291-304.

Mucchielli, Laurent (2003): Délinquance et immigration en France : un regardsociologique. In: Criminologie, 36, 2, S. 27-55

Mucchielli, Laurent/Lapeyronnie, Didier (2005): Grosse, leereWorte. In: Berliner Zeitung, 10.11.05,S. 32.

Pager, Devah (2008): The Republican ideal? National minorities and the criminal justice system in contemporary France. In: Punishment\& Society, 10(4), S. 375-401.

Pruvost, Geneviève (2009): Living and working conditions in the police in 2003. A sociologicalsurvey. In: Penal Issue, Juni 2009.

Pruvost, Geneviève/RoharikIonela (2011): Comment devient-on policier ? 1982-2003.

Évolutionssociodémographiques et motivations plurielles. In: Déviance et Société, 35, 3, S. 281 312 .

Safi, Mirna/Simon, Patrick (2013): Les discriminations ethniques et racialesdansl'enquêteTrajectoires et Origines: Représentations, expériencessubjectives et situations vécues. In: Economie et Statistique, 464-466, S. 245-274.

Schnapper, Dominique (2008): Les enjeuxdémocratiques de la statistiqueethnique. In: RevueFrançaise de Sociologie, 49-1, S. 133-39.

Simon, Patrick (2008): The choice of ignorance: the debate on ethnic and racial statistics in France. In: French Politics, Culture \& Society, 26-1, S.7-31.

Tournier, Pierre/Robert, Philippe (1991):Etrangers et délinquances: les chiffres du débat. Paris : L'Harmattan.

Wieviorka, M. (Hg.), (1992):La France raciste. Paris: Le Seuil.

Zauberman, Renée(1998): Gendarmerie et gens du voyage en régionparisienne. In: Cahiersinternationaux de sociologie, 55, S. 415-452.

Zauberman, Renée \& Lévy, René (2003): Police, Minorities and the French Republican Ideal. In: Criminology, 41, 4, S. 1065-1100. 